\title{
THE USE OF 3D PRINTING IN MANUFACTURING ANTHROPOMORPHIC PHANTOMS FOR BIOMEDICAL APPLICATIONS
}

\author{
Kristina Bliznakova \\ Laboratory of Computer Simulations in Medicine, Technical University of Varna
}

\begin{abstract}
Emerging imaging modalities require urgently the use of physical anthropomorphic phantoms. They are used to design, test and evaluate the performance of the new forthcoming imaging systems, prior to their introduction into clinical practice. However, until recently, manufacturing of such phantoms was challenging due to limitations in the current technology, suitable materials, manufacturing precision, and size of digital models. This paper presents the application of three-dimensional (3D) printing in the field of biomedical engineering. Specifically, the focus is on the development of anthropomorphic physical phantoms with characteristics suitable for use in $x$-ray imaging. The use of 3D printing in the educational process of engineers, doctors and physicists is also demonstrated.
\end{abstract}

Keywords: 3D printing, biomedical applications, research and education, breast phantoms

\section{INTRODUCTION}

The physical anthropomorphic phantoms play a critical role in the contemporary development of diagnostic radiology. They permit safe unlimited exposure and are intended for use in image quality and patient dose optimization, clinical commissioning and pre-clinical trials in health technology assessments. One of the key applications of the physical anthropomorphic phantoms is in the field of testing, evaluation and clarifying the role and the benefits of newly developed imaging modalities. For example in the field of x-ray breast imaging, these are breast tomosynthesis, breast computed tomography and dual-energy breast imaging. Development of anthropo-

Address for correspondence:

Kristina Bliznakova

Laboratory of Computer Simulations in Medicine

Technical University of Varna

1 Studentska Str

9010 Varna, Bulgaria

e-mail: kristina.bliznakova@gmail.com

Received: April 28, 2015

Accepted: May 14, 2016 morphic physical three-dimensional (3D) phantoms with a realistic tissue distribution are necessary to optimize settings such as incident $\mathrm{x}$-ray spectra, detectors, acquisition geometries, time for scanning as well as testing and optimizing image reconstruction techniques.

Manufacturing of very realistic anthropomorphic phantoms, however, is a challenging task. It is associated with difficulties, such as the current 3D printing technology, the limited number of available materials for printing, which in most cases do not properly reflect the properties of the human tissues, manufacturing precision, limitations in the size of the printed model and its resolution, format of the software models, etc. Due to these shortcomings, there is a very limited number of anthropomorphic physical phantoms either available or currently under development for studies in x-ray imaging (1-5). There is a strong need for further research and technology improvement in this field.

The aim of this paper is to present the use of 3D printers for biomedical applications. Examples of application of 3D printing are selected from the field of x-ray imaging. These examples are taken from 
the research work of the Biomedical Engineering group from the Technical University of Varna, Bulgaria, which research is dedicated to developing, testing and optimising novel $\mathrm{x}$-ray breast imaging techniques. The rest of the paper is structured as follows: in Section II, the basic 3D printing technologies are shortly described; examples of 3D printing with stereolithography and fused deposition technology are given in Section III; applications of 3D printing for biomedical applications are listed in Section IV. Finally, conclusions are drawn on the future perspective of the application of 3D printing technologies for novel x-ray diagnostic techniques.

\section{D Printing Technologies}

$3 \mathrm{D}$ printing is a prototyping process, in which a real object is created from a 3D digital model. The 3D printers create the objects layer by layer and this process is known as desktop fabrication or additive manufacturing. The digital 3D model is saved in a common file format (usually STL) and then transmitted to the $3 \mathrm{D}$ printer for printing.

The printing resolution of current printers is between $328 \times 328 \times 606$ DPI (xyz) and 656 x 656 x 800 DPI (xyz) in ultra-HD resolution, while the corresponding voxel size ranges between $0.025 \mathrm{~mm}$ and $0.05 \mathrm{~mm}$. The dimensions of the printed objects may reach values of up to $1000 \mathrm{~mm}$ along the $\mathrm{x}, \mathrm{y}, \mathrm{z}$ directions. There are several technologies used to print models, which mainly differ in the way the printing layers are built to create parts. This results in different properties of the final printed 3D objects. Among the most famous technologies dedicated to 3D printing are: Selective Laser Sintering (SLS), Stereolithography (SLA) and Fused Deposition Modelling (FDM). These technologies are schematically shown in Figure 1.

In the SLS technology for fast prototyping (Figure 1a), the 3D printed model is printed from powders, such as wax, polystyrene, nylon, glass, ceramics, stainless steel, titanium, aluminium, or various alloys. The printed objects are lightweight. The printing process starts with cutting the $3 \mathrm{D}$ digital model into thin layers. Each layer is then sent to the 3D printing device. There are two main parts in the SLS technology: a container filled with powder polymer and a device, which is used to distribute the powder in the form of thin layers, as shown in Figure 1a. During the $3 \mathrm{D}$ printing process, a fine layer of powder is laid down and then using a laser controlled by the software of the $3 \mathrm{D}$ printing device, some of the powder granules are selectively fused together. These are granules the position of which corresponds to the position of the computer model. When the current layer is printed, the construction platform is lowered, and a new thin layer of powder is deposited on the printing model and the process is repeated until the prototype is completed. During printing, non-bonded powder granules support the object as it is being constructed. Once printing is completed, almost all of the excess powder can be recycled.

The SLA technology produces very small and fine details with perfectly smoothed surfaces, which

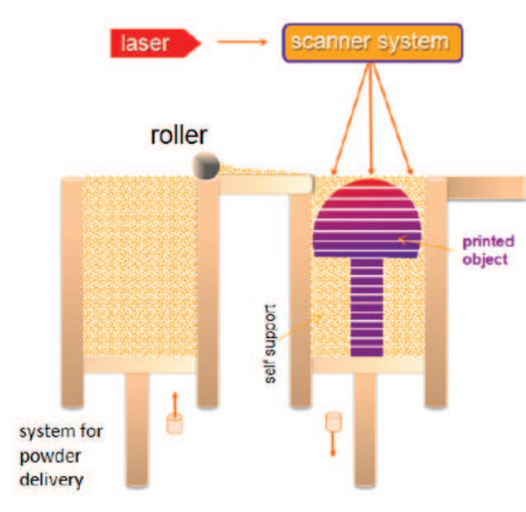

(a)

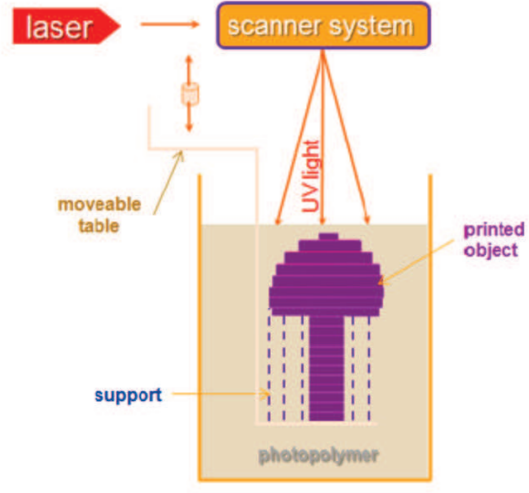

(b)

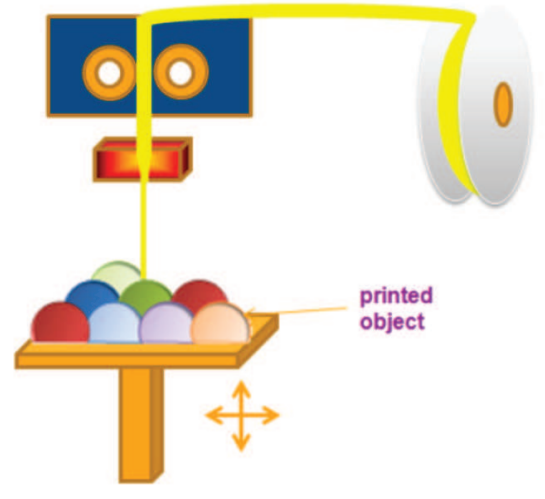

(c)

Figure 1. Schematic presentation of the most commonly used 3D printing technologies: (a) Selective Laser Sintering (SLS), (b) Stereolithography (SLA) and (c) Fused Deposition Modelling (FDM). 
do not normally require any additional processing. The minimum built layer thickness of such 3D printer may reach $0.02 \mathrm{~mm}$. Stereolithography 3D printers position a moving base/platform just below the surface of a vat of liquid photocurable polymer/resin (Figure 1b). An ultraviolet laser beam then traces the first layer of the printed object on the surface of the liquid polymer, causing a very thin layer of photo-induced polymer to harden. The base is then lowered or raised slightly (depending on the type of the printer) and another layer is traced out, hardened by the laser, and produced. This process continues until the complete object is built up. Then the object is removed from the vat of photopolymer, drained of excess liquid, and cleaned. The SLA printers remain among the most accurate ones for fabrication of $3 \mathrm{D}$ objects.

In the FDM technology, the 3D printed object is created layer by layer through heating the thermoplastic material to a semi-liquid state, and extrusion and deposition of the material, according to a computer model (Figure 1c). FDM may use more than one material to accomplish the process: e.g. one material for the printed object itself, and another auxiliary material, either for the printed object again (to create objects of two or more different materials), or a material that is soluble and may be used as a base or a supporting structure. Objects are produced with a high degree of accuracy. Most FDM 3D printers can print with acrylonitrile butadiene styrene (ABS), biodegradable bioplastic called PLA-polylactic acid, which is produced from organic alternatives of oil.

\section{D Printing with FDM and SLA}

The Biomedical Engineering group at the Technical University of Varna uses two 3D printers: the first one is based on the FDM technology (LeapFrog Creatr 3D printer - dual head ABS printer) and the second one is based on the SLA technology (FORM 1+, FormLabs, Somerville, MA). These printers are used daily in preparing different phantoms dedicated to research and educational purposes in the field of x-ray imaging. Some of the characteristics of the two printers are listed in Table 1.

These printers were tested to produce two test objects of particular interest for the research work of the team. One of the objects was a compressed breast phantom, shown in Figure 2a,b, and the other one was a dental construction shown in Figure 2c,d. In Figure $2 a, c$, the objects are printed with the SLA printer, while in Figure 2b,d the objects are printed with the FDM printer. As seen from these images, the objects printed with the SLA technology are characterized by smooth surfaces compared to the objects printed with the FDM one. Both models are printed with a $0.1 \mathrm{~mm}$ layer thickness. Moreover, the samples with the SLA printer are printed using Clear resin material. The objects in Figure 2a,b are used in studies of $\mathrm{x}$-ray breast imaging techniques, while the dental constructions are used in a collaborative study with the Faculty of Dental Medicine, Medical University of Varna, Bulgaria (6-8).

Speed and temperature are key factors in the 3D printing process. Failing to correctly choose one of these parameters may result in defective printed objects, as demonstrated in Figure 3.

Table 1. Details for the printers used in the daily work at TU-Varna

\begin{tabular}{lcc}
\hline Criterion & FDM & SLA \\
Resolution of the printed object & $50 \mu \mathrm{m}$ & $25 \mu \mathrm{m}$ \\
STL file size & no limit & $<100 \mathrm{MB}$ \\
Printing speed & slow-to-average & average-to-fast \\
Price of the printer & $\sim 2000$ USD & $\sim 2800$ EUR \\
Cost for the supplies & Low cost & Average cost \\
Materials for printing & ABS discs & Resin \\
Software & paid software license & free of charge software license \\
Size of the printed object & $230 \times 260 \times 200, \mathrm{~mm}$ & $125 \times 125 \times 165$, mm \\
\hline
\end{tabular}

Scripta Scientifica Medicinae Dentalis, vol. 2, No 1, 2016, pp. 23-31 


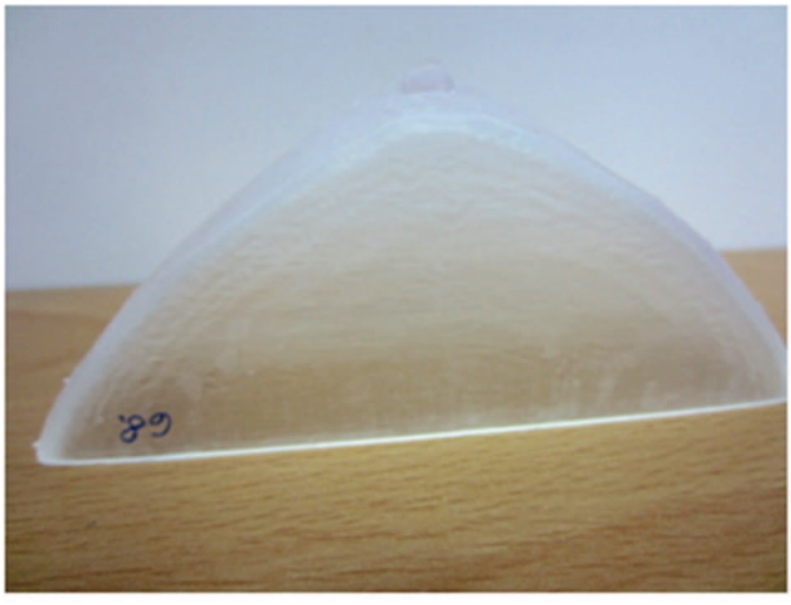

(a)

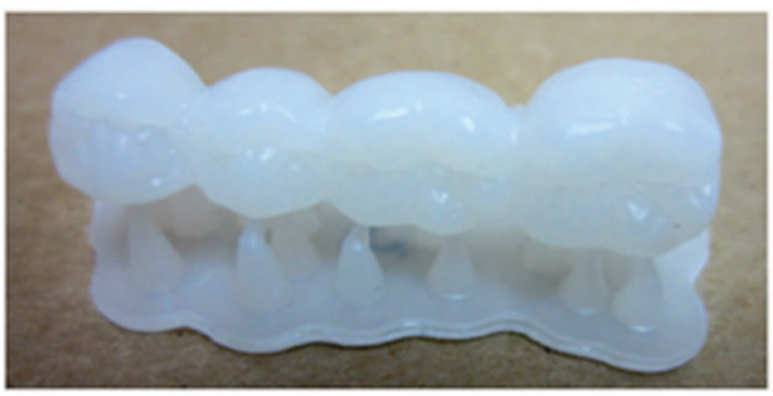

(c)

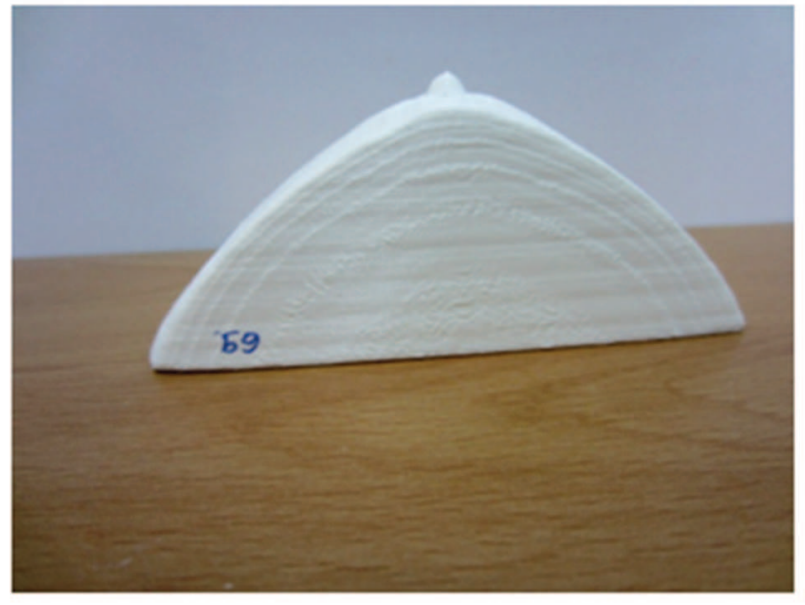

(b)

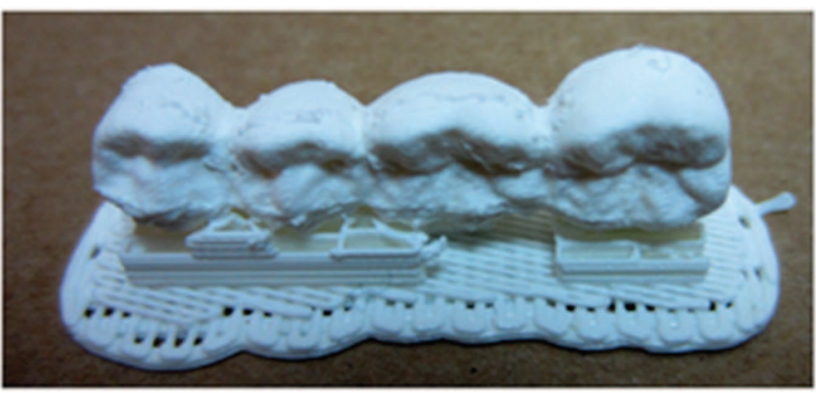

(d)

Figure 2. Test objects printed with SLA $(a, c)$ and FDM $(b, d) 3 D$ printers

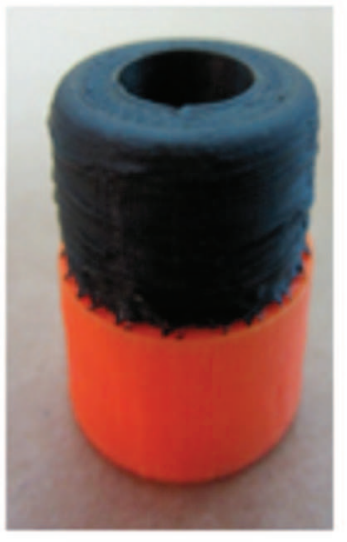

(a)

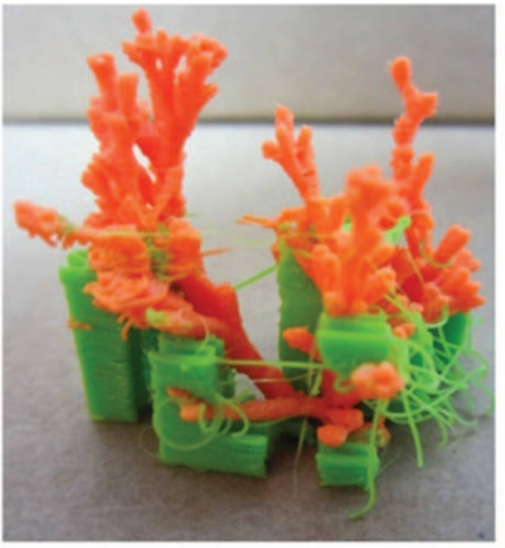

(b)

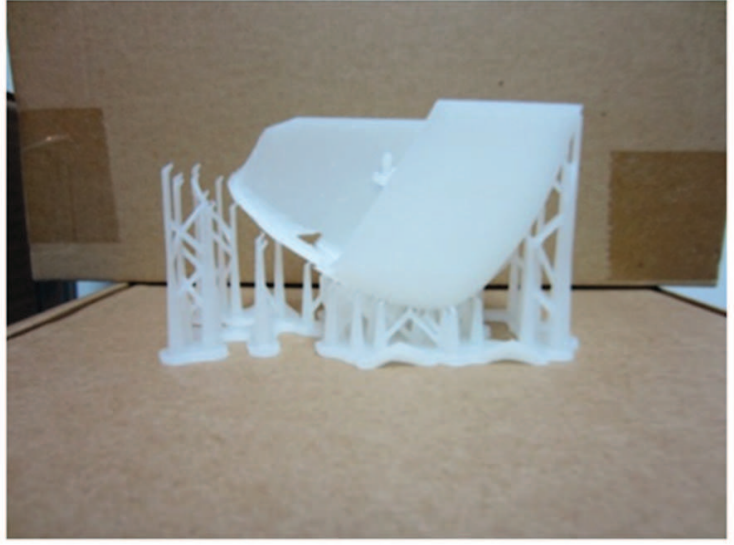

(c)

Figure 3. Examples of defective print-outs due to incorrect printing process settings 
For the test object printed in Figure 3a, the defective printing was due to both unsuitable speed and temperature (mainly temperature) selected for the specific material. Figure $3 \mathrm{~b}$ shows a trial for printing a duct tree of the breast phantom with two ABS materials simultaneously. The parameters for this type of printing process are still under optimization. Defective printing with the SLA printer is demonstrated in Figure 3c. The reason for this defect is under clarification. cluded ultrasound measurements. It is worth saying that there currently are no ultrasonic phantoms that mimic in a realistic fashion the structure of trabecular bone. The use of 3D printing opens new horizons. Figure $4 \mathrm{~b}$ shows three different adult anthropomorphic phantoms printed at the University of Florida (1). These phantoms are dedicated to x-ray dosimetry studies. They are constructed in $5 \mathrm{~mm}$ transverse slices using materials designed to mimic human tissue at diagnostic photon energies, like urethane-

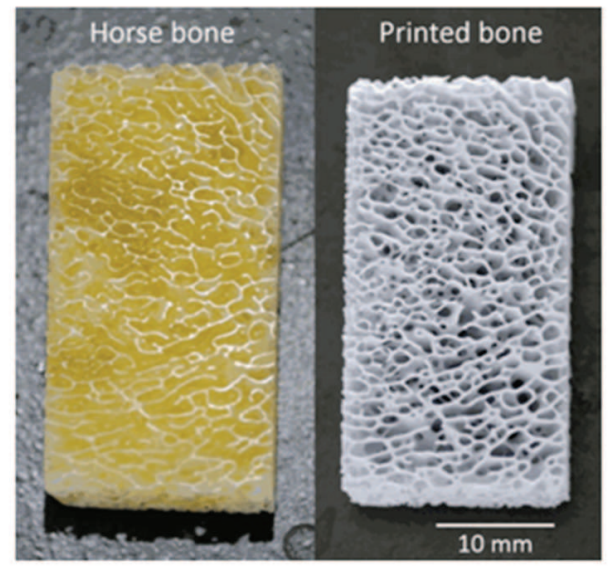

(a)

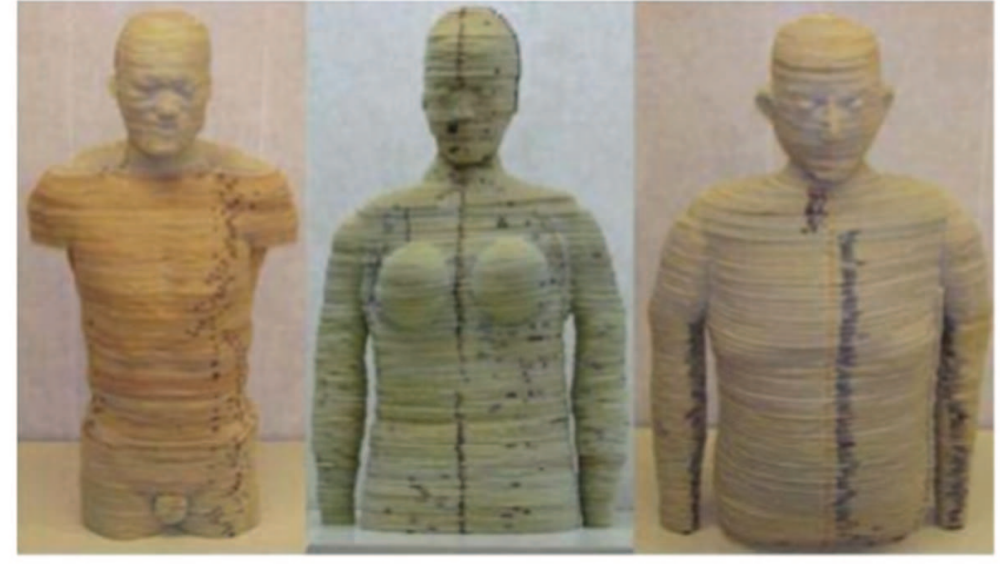

(b)

Figure 4. Examples of printed anthropomorphic phantoms for applications in biomedical science. (a) Comparison between trabecular horse bone - real (left) and 3D printed (right), Mézière F et al. (9). Reprinted with the kind permission of the publisher AIP Publishing LLC; (b) phantoms based on a segmented CT data set of an adult male (left), phantom based on a computational adult female hybrid data set (middle), phantom based on a computational adult male hybrid data set (right), Winslow J et al: (1). Reprinted with the kind permission of the author

\section{Applications of 3D Printing for Biomedical Applications}

During the last few years, the application of $3 \mathrm{D}$ printing in biomedical research has grown enormously. A basic requirement is the digital (software) model. Such models may be obtained from, either 3D imaging techniques such as MRI, CT, PET, etc., or dedicated CAD software.

Such an example is demonstrated in Figure 4a, where a micro-structure of a trabecular horse bone sample was obtained via synchrotron $\mathrm{x}$-ray microtomography, and then converted to a 3D binary data set, and successfully 3D-printed at scale 1:1 (9). The phantom was printed with an SLA printer. Further, this phantom was used in experimental work in- based compound. The phantom slices are based on original segmented computed tomography patient datasets.

In all these applications, the main approach from the digital model to the printed model may be summarized as shown in Figure 5. The process is comprised of several main steps, required for printing the physical phantoms: (a) obtaining the digital model, (b) obtaining the CAD model; (c) additional image processing techniques in order to smooth the surface of the object, to remove the pixelization, (d) obtaining the STL format of the printed object, (e) printing the object itself, and (f) applying additional post-processing of the printed model. 


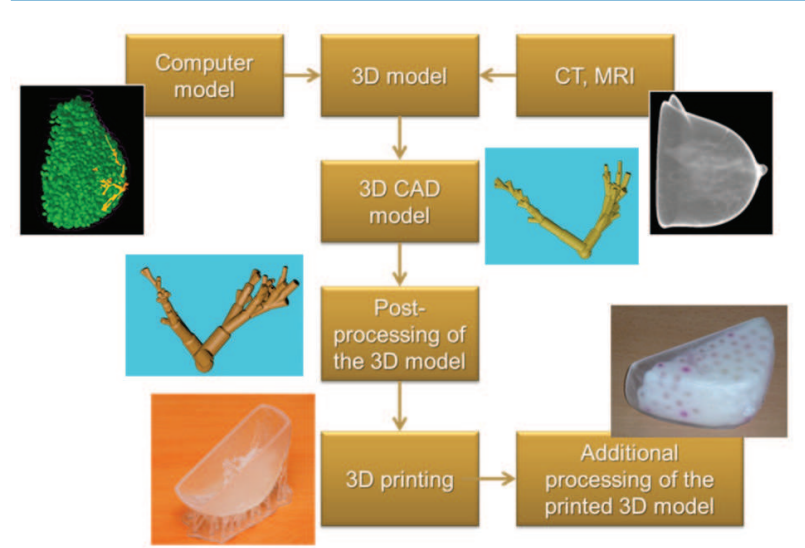

Figure 5. Flow-chart diagram of the presented approach for $3 D$ printing of phantoms dedicated to $x$-ray imaging images acquired from $3 \mathrm{D}$ diagnostic techniques. One such example is demonstrated in Figure 6 for the case of the liver. The liver shape is automatically selected (12) from patient abdominal CT images and consequently, the region that belongs to the liver was segmented from the other tissues and used in preparing the final 3D liver volume.

Once the digital phantom is created, the $3 \mathrm{D}$ CAD model is prepared. This requires the implementation of several image-processing techniques which aim at converting the model to a model comprised of one or two tissues only. This is imposed by the fact that most of the low budget printers print with one or

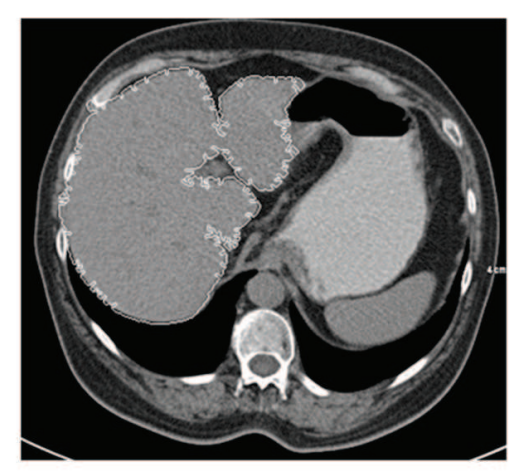

(a)

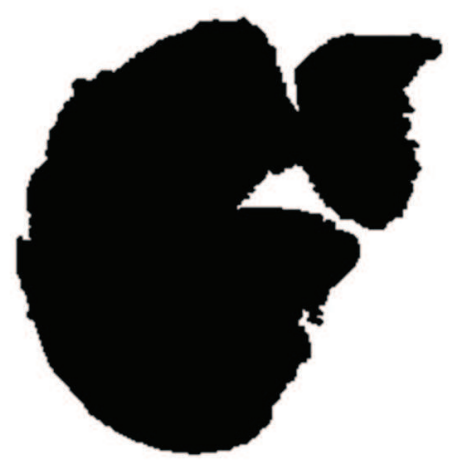

(b)

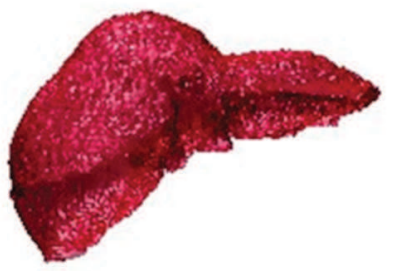

(c)

Figure 6. Modelling of computational objects from patient-based CT images: (a) a CT image extracted from a patient scan, (b) segmented liver, (c) $3 D$ liver for printing

This process is described in details for the specific case of breast phantoms, which are produced with the 3D printers available at the Biomedical Engineering group, Technical University of Varna. At first, a computer model of a breast digital phantom is created. This phantom may be obtained either by dedicated software, or by patient specific data, acquired through one of the 3D diagnostic techniques: MRI, CT, PET, etc. which provide 3D imaging data. In the example, demonstrated in Figure 5, the computational model of the breast is designed with an inhouse developed software $(10,11)$, dedicated to generation of complete computer based realistic breast models for x-ray imaging and their use in virtual experiments that aim at design, testing and optimizing of new $\mathrm{x}$-ray breast imaging techniques. The second possibility is to create $3 \mathrm{D}$ computational objects from two materials simultaneously. In this way the printing material may be used to reflect the properties of one of the tissues, while the second one, called filling material may be used to approximate another tissue. For example, in the case of Figure 5, the ABS/ resin material is used as a tissue-equivalent material for the glandular part of the breast as well as the skin, while the animal fat may be used to fill the printed volume and to approximate the adipose tissue of the breast.

The third step is related to applying image-processing operations on the voxel-based volume (Figure 7a). These mainly include filtering techniques that allow smoothing of the voxel-based model (Figure $7 \mathrm{~b}$ ) and decreasing the possible artefacts from the pixelization in the final printed version. This pixel- 


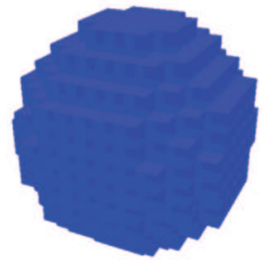

(a)

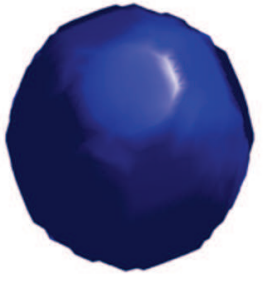

(b)

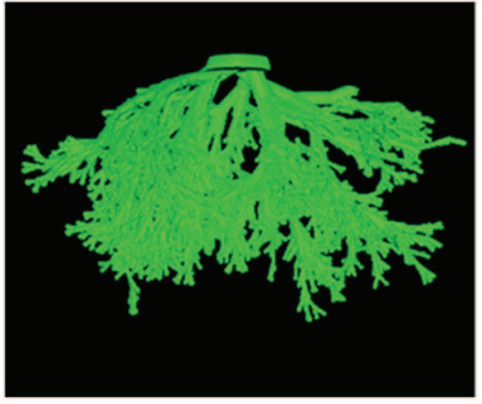

(c)

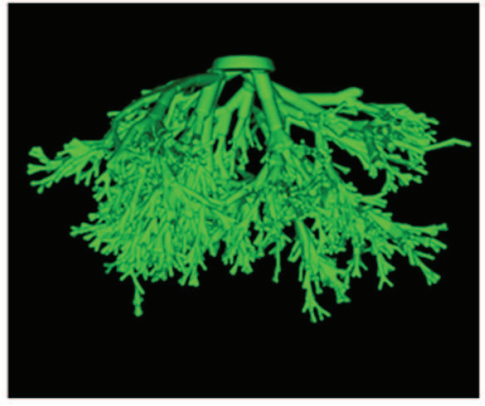

(d)

Figure 7. Additional image processing applied on the digital model aiming at smoothing the surface of the printed object: $(a, c)$ original model, $(b, d)$ processed model after smoothing

ization is due to the size of the voxels in the software model. As seen in Figure 7, applying the smooth operation on the original voxel-based version (Figure 7c) results in a smoother object version (Figure 7d).

The step before printing is writing the model in an STL file format. This format is supported by the majority of software packages for rapid prototyping. Once printed, the objects are subjected to additional post-processing, such as removing the support structures and using different materials to complete the final object.

Examples of 3D Printing Implemented at the Technical University of Varna

$3 \mathrm{D}$ printing is currently the main tool in the research of the Biomedical Engineering group at Technical University of Varna, whose research is oriented towards development, testing and optimising of novel $\mathrm{x}$-ray breast imaging techniques (www.maxima-tuv.eu). This research is provoked by the fact that although technology has advanced in the last years, breast cancer is by far the most common form of cancer for women under seventy years of age with an estimated 1.4 million new cancer cases diagnosed in 2012 (23\% of all cancers). Emerging breast x-ray imaging modalities have the potential to improve early diagnosis of breast cancer. These are breast tomosynthesis, breast $\mathrm{CT}$, and phase-contrast breast imaging. However, they must be well validated, before being used in clinical practice. Currently, most of the physical phantoms for x-ray breast imaging are produced in simple geometrical forms, made of homogeneous background with introduced test objects that approximate lesions, like the tissue equivalent phantom for mammography (CIRS 010A) Figure 8a,b. In practice, however, it is known that cancer margins are not well outlined and cannot be separated from the healthy tissues. Therefore, these phantoms are not appropriate for research on cancer detection. In addition, due to the homogeneous background of these phantoms, their use is limited to dose measurement and quality control procedures only, as can be seen in Figure 8c. Realistic physical breast models with breast abnormalities with irregular shapes are needed. Such models are designed and printed at Technical University of Varna. These are shown in Figure 8d,e, while Figure $8 \mathrm{f}$ shows a CT slice from the scanned phantom, shown in Figure 8e.

Besides research, 3D finds active application in educational issues. 3D printing was a practical exercise of the "Anthropomorphic Phantoms" module, which is a part of the EUTEMPE-RX course: European Training and Education for Medical Physics Experts in Radiology (www.eutempe-rx.org), held at the Technical University of Varna, Sept 7-13, 2015. Participants were encouraged to develop skills for the design and evaluation of anthropomorphic phantoms, as well as to design, manage, implement and evaluate virtual clinical studies with such phantoms, and to discuss and interpret the results of the virtual studies. 3D printing was used during the third day of the course, in teaching the subject "Computational Anthropomorphic Phantoms as Prototypes of Physical Objects".

During this subject, participants designed their own digital breast phantoms, processed them properly, worked with the dedicated software for import- 


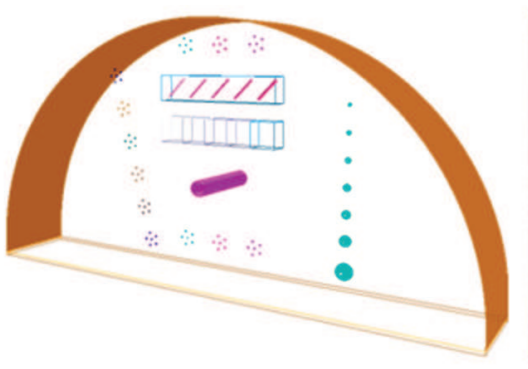

(a)

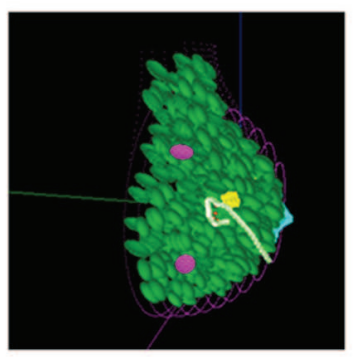

(d)

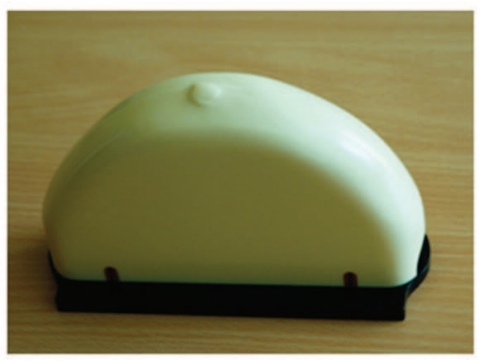

(b)

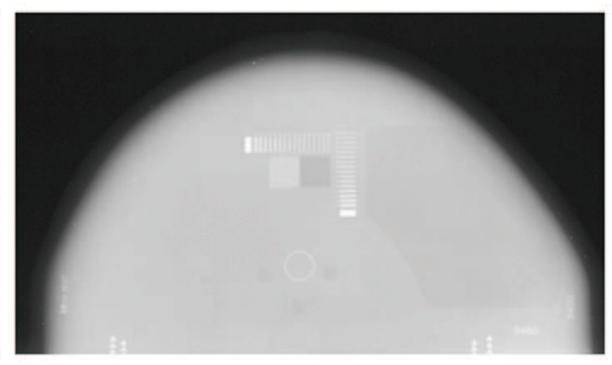

(c)

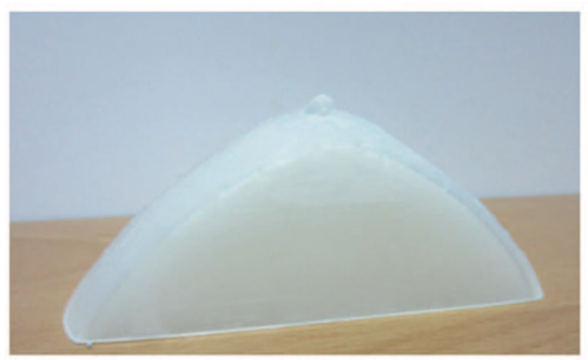

(e)

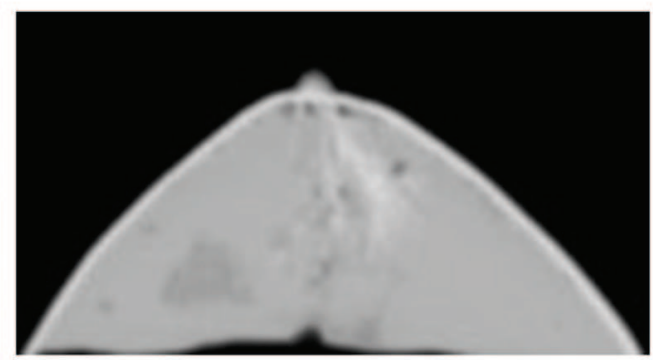

(f)

Figure 8. Commercial CIRS 010A tissue equivalent phantom $(a, b, c)$ and in-house $(d, e, f)$ breast physical phantom developed at TU-Varna. ( $a, d)$ digital phantom, (b,e) physical phantom; (c) x-ray mammography and $(f)$ a CT image of the corresponding phantoms. The mammography image (c) is kindly provided by Prof. Paolo Russo, University of Naples -

Federico II, Italy

ing STL files and adding different support structures, and finally to print the models. Results of successfully generated and printed computational models of the breast are demonstrated in Figure 9.

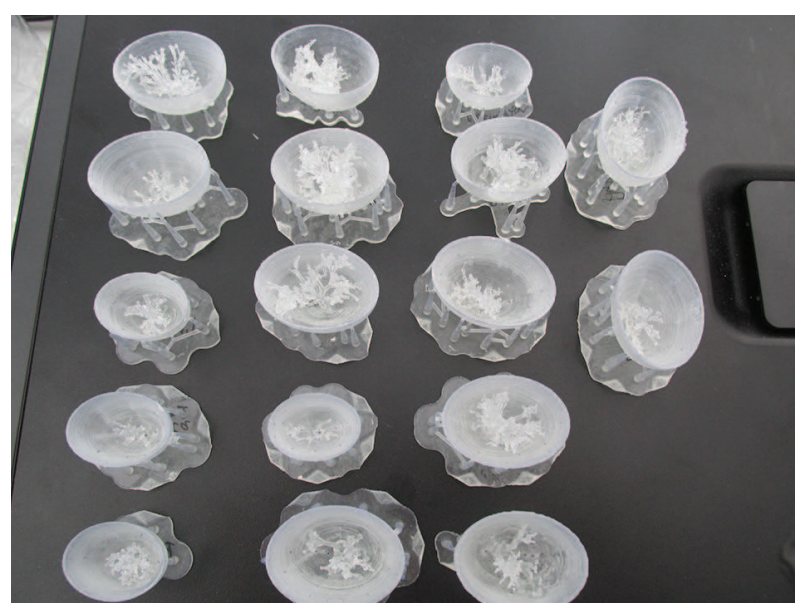

Figure 9. Printed objects by the EUTEMPE-RX participants

\section{CONCLUSIONS}

The future development of new diagnostic and treatment techniques, their testing and optimisa- tion is highly dependent on the development of the $3 \mathrm{D}$ printing technology. Nowadays, most of the modern radiology departments own their own 3D printers used to manufacture different physical phantoms for validation and optimisation of specific imaging protocol. The future, when patient specific physical phantoms will be daily printed and the treatment of patients will be based on their own physical phantoms, is not far. The availability of such models is a powerful instrument in the hands of engineers, physicists and physicians to be used in the development of new technologies for precise definition of the boundaries of these cancers. 3D characterization of the cancer is of major help in determining the recalls from the screening and in the pre-operative work up of cancers. There is no doubt that the further development of 3D printing technology will speed up further progress in this direction.

\section{ACKNOWLEDGEMENTS}

The paper was presented at workshop-demonstration " $3 D$ printing - peculiarities, application and perspectives" which was held 27-28 Nov. 2015 at Faculty of Dental Medicine, Medical University of Varna, Bulgaria. The workshop was supported by the project 
with contract 502/19, 12 Dec 2014, of the Fund for Scientific Investigations, Ministry of Education and Science of Bulgaria.

\section{REFERENCES}

1. Winslow, J.F., et al., Construction of anthropomorphic phantoms for use in dosimetry studies. Journal of Applied Clinical Medical Physics, 2009. 10(3): p. 195-204.

2. Kiarashi, N., et al., Development of realistic physical breast phantoms matched to virtual breast phantoms based on human subject data. Med Phys, 2015. 42(7): p. 4116-26.

3. Carton, A.K., et al. Development of a 3D high-resolution physical anthropomorphic breast phantom. in Medical Imaging 2010: Physics of Medical Imaging. 2010. San Diego, CA.

4. Leng, S., et al., Construction of Realistic Liver Phantoms from Patient Images using 3D Printer and Its Application in CT Image Quality Assessment. Medical Imaging 2015: Physics of Medical Imaging, 2015. 9412.

5. Anderson, J.R., et al., Validation of computational fluid dynamics methods with anatomically exact, 3D printed MRI phantoms and 4D pcMRI. Conf Proc IEEE Eng Med Biol Soc, 2014. 2014: p. 6699-701.

6. Dikova Ts., Dzhendov D., Simov M., Katreva-Bozukova I., Angelova Sv., Pavlova D., Abadzhiev M., Tonchev Ts., 2015, Modern trends in the development of the technologies for production of dental constructions, Journal of IMAB-Annual Proceeding Scientific Papers, 21(4), pp.974-981.

7. Dikova Ts., Dzhendov D., Katreva I., Pavlova D., Simov M., Angelova Sv., Abadzhiev M., Tonchev Ts., 2016, Possibilities of 3D printer Rapidshare D30 for manufacturing of cube samples, Scripta Scientifica Medicinae Dentalis, vol. 2, No 1, 2016, p.9-15

8. Katreva I., Dikova Ts., Abadzhiev M., Tonchev Ts., Dzhendov D., Simov M., Angelova Sv., Pavlova D., Doychinova M., 3D-printing in contemporary prosthodonthic treatment, Scripta Scientifica Medicinae Dentalis, vol. 2, No 1, 2016, p.17-22

9. Meziere, F., et al., Experimental observation of ultrasound fast and slow waves through three-dimensional printed trabecular bone phantoms. J Acoust Soc Am, 2016. 139(2): p. EL13.

10. Bliznakova, K., et al., Breast Simulator: A software platform for breast x-ray imaging research. Jour- nal of Biomedical Graphics and Computing, 2012. 2(1): p. 1-14.

11. Bliznakova, K., et al., Evaluation of an improved algorithm for producing realistic 3D breast software phantoms: Application for mammography. Med Phys, 2010. 37(11): p. 5604-5617.

12. Bliznakova, K., et al., Computer aided preoperative evaluation of the residual liver volume using computed tomography images. J Digit Imaging, 2015. 28(2): p. 231-9. 